\title{
Underreporting of Cases During the COVID-19 Pandemic: A Worrying Warning for Africa
}

\author{
Marcos Roberto Tovani-Palone ${ }^{1 *}$ (), Federico Garoli ${ }^{2}$ (D), Pritik A Shah ${ }^{3}$ (D), Manar Ahmed Kamal ${ }^{4}(\mathbb{D}$, \\ Faisal A Nawaz ${ }^{5}$ (1)
}

\author{
${ }^{1}$ Ribeirão Preto Medical School, University of São Paulo, Ribeirão Preto, BRAZIL \\ ${ }^{2}$ Department of Biomedical Sciences, Humanitas University, Milan, ITALY \\ ${ }^{3}$ Bangalore Medical College and Research Institute, Bangalore, Karnataka, INDIA \\ ${ }^{4}$ Faculty of Medicine, Benha University, Benha, EGYPT \\ ${ }^{5}$ College of Medicine, Mohammed Bin Rashid University of Medicine and Health Sciences, Dubai, UNITED ARAB EMIRATES \\ *Corresponding Author: marcos_palone@hotmail.com
}

Citation: Tovani-Palone MR, Garoli F, Shah PA, Kamal MA, Nawaz FA. Underreporting of Cases During the COVID-19 Pandemic: A Worrying Warning for Africa. Journal of Contemporary Studies in Epidemiology and Public Health. 2022;3(1):ep22001. https://doi.org/10.30935/jconseph/11467

\begin{abstract} Africa, its implications, as well as propose recommendations.

Keywords: pandemics, COVID-19, Africa, public health surveillance, epidemiology

Received: 8 Nov. $2021 \bullet$ Accepted: 26 Nov. 2021
\end{abstract}

In this manuscript, we discuss the current epidemiological scenario of coronavirus disease 2019 (COVID-19) in

Dear Editor,

According to the World Health Organization (WHO), there is a severe underestimation of Coronavirus disease 2019 (COVID-19) cases in Africa [1]. A recent assessment shows that only one in seven cases of COVID-19 (14.2\% of the total) have been reported in the region. The possible cause for this problem has probably been the low number of COVID-19 tests performed, mostly including patients with overt clinical symptoms and international travelers.

It is estimated that about 59 million Africans were affected by the severe acute respiratory syndrome Coronavirus 2 (SARS-CoV-2); however, there are only 8.5 million officially reported cases. So far, a total of 70 million COVID-19 tests were carried out on a population of 1.3 billion, which highlights the significant gap in reported cases. In response to this, WHO has focused on increasing COVID-19 testing capacity. The countries included are Burundi, Cote d'Ivoire, the Democratic Republic of the Congo, Guinea-Bissau, Mozambique, Republic of Congo, Senegal, and Zambia [1].

Monitoring the COVID-19 pandemic's impact in Africa has been a considerable challenge, owing to relatively weak health services that cannot execute containment tactics and lack of data to track the situation [2, 3]. However, even if we had more data on monitoring the epidemic across the continent over time, the overall repercussions are now clearly anticipated without the need for additional modeling studies [1]. Furthermore, because the pandemic degrades the global economy, an increase in absolute poverty is expected, putting the Sustainable Development Goals (SDGs) in jeopardy with catastrophic consequences for the African continent [4]. The flowchart shown in Figure 1 summarizes the consequences of underreporting.

In the current situation, all stakeholders must work together to devise measures for limiting the scope of the current extended emergency without jeopardizing the delivery of primary health care. Strengthening the health system might boost the availability of reliable data and contribute more effectively to combat the pandemic's severe repercussions. More than ever, practical assistance and strong partnership across international organizations are required.

Moreover, given the potential severity of the disease [5-7], the circulation of SARS-CoV-2 variants, and the low vaccination rates against COVID-19 across the African territory [8], it is imminent that governments and health authorities strengthen local surveillance actions [9]. Increased funding towards COVID-19 screening centers may further guarantee access to appropriate treatment and preventative health services in response to the high-cost supplies for the management of severe cases $[10,11]$. Further research is warranted in understanding the socio-geographical factors and the role of public hesitancy to accessing healthcare, which may negatively influence COVID-19 detection and vaccination rates in the region.

In this context, the underestimated burden of long-lasting COVID19 sequelae and complications requires extensive efforts in restructuring the allocated funding, resources and infrastructure for the African health system $[12,13]$. Failure to mitigate this hidden crisis can derail the path to global recovery and sustainability of the continent. Through international collaborations, funding support, and research focused on efficient reporting of cases, this public health challenge can 


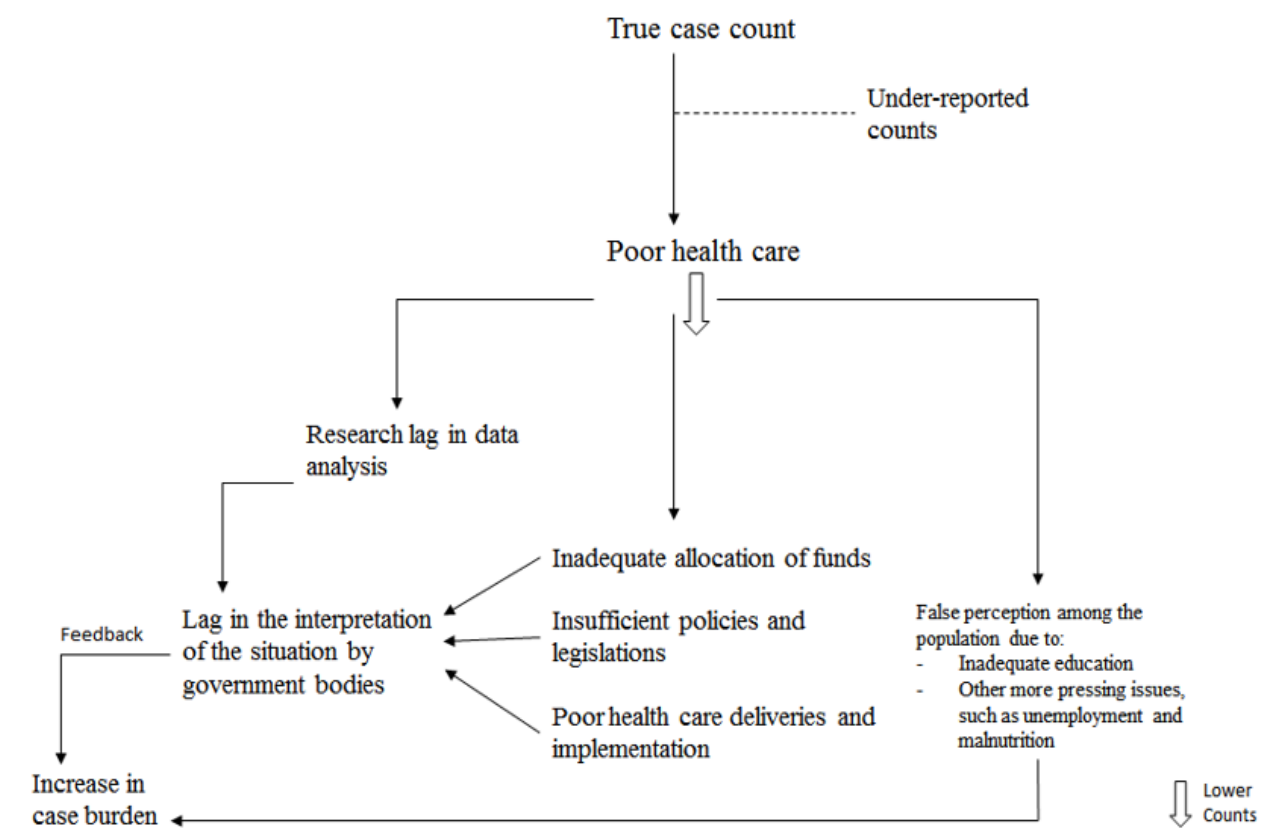

Figure 1. Schematic representation of the consequences of the underreporting of COVID-19 cases in Africa (cause and effect analysis)

serve as an evidence-based incubator for innovation during the pandemic.

Author contributions: All authors were involved in concept, design, collection of data, interpretation, writing, and critically revising the article. All authors approve final version of the article.

Funding: The authors received no financial support for the research and/or authorship of this article.

Declaration of interest: Authors declare no competing interest.

Data availability: Data generated or analysed during this study are available from the authors on request.

\section{REFERENCES}

1. World Health Organization (WHO). Six in seven COVID-19 infections go undetected in Africa. Available at: https://www.afro.who.int/news/six-seven-covid-19-infectionsgo-undetected-africa (Accessed: 17 October 2021).

2. Post LA, Argaw ST, Jones C, et al. A SARS-CoV-2 surveillance system in sub-Saharan Africa: modeling study for persistence and tansmission to inform policy. J Med Internet Res. 2020; 22(11): e24248. (doi: 10.2196/24248). PMID: 33211026; PMCID: PMC7683024.

3. Fai KN, Corine TM, Bebell LM, et al. Serologic response to SARSCoV-2 in an African population. Sci Afr. 2021; 12: e00802. (doi: 10.1016/j.sciaf.2021.e00802). PMID: 34095639; PMCID: PMC8164732.
4. Odey GO, Alawad AGA, Atieno OS, et al. COVID-19 pandemic: impacts on the achievements of sustainable development goals in Africa. Pan Afr Med J. 2021; 38: 251. (doi: 10.11604/pamj.2021.38.251.27065). PMID: 34104299; PMCID: PMC8164424.

5. Khawaja UA, Shamsoddin E, Desideri LF, Tovani-Palone MR. Infection of red blood cells by SARS-CoV-2: new evidence. Einstein (Sao Paulo). 2021; 19: eCE6285. (doi: 10.31744/einstein_journal/2021CE6285). PMID: 33886935; PMCID: PMC8029641.

6. Giacalone M, Tovani-Palone MR, Marin L, Febbi M, Russano T, Giacalone A. Neurological and neuropsychiatric disorders associated with COVID-19. Part I: overview and neurological disorders. Einstein (São Paulo). 2021; 19: eCE6448. (doi: 10.31744/einstein_journal/2021CE6448). PMID: 34730705; PMCID: PMC8528447.

7. Tovani-Palone MR, Storari L, Barbari V. Could preventing thromboembolism be a new hope for COVID-19 patients? Electron J Gen Med. 2020; 17(6): em250. (doi: 10.29333/ejgm/8244).

8. Uwishema $\mathrm{O}$, Onyeaka $\mathrm{H}$, Alshareif BAA, et al. Current context of pneumonia amidst the COVID-19 pandemic in Africa. J Contemp Stud Epidemiol Public Health. 2021; 2(1): ep21007. (doi: 10.30935/jconseph/11281). 
9. Tovani-Palone MR, Siddiqui MF. COVID-19 surveillance of healthcare workers during the SARS-CoV-2 vaccination period: an updated protocol suggestion. Clinics (Sao Paulo). 2021; 76: e3096. (doi: 10.6061/clinics/2021/e3096). PMID: 34190857; PMCID: PMC8221565.

10. Pedersini P, Villafañe JH, Corbellini C, Tovani-Palone MR. COVID-19 pandemic: a physiotherapy update. Electron J Gen Med. 2021; 18(1): em264. (doi: https://doi.org/10.29333/ejgm/8574).

11. Tovani-Palone MR, Shamsoddin E. Use of mouthwashes in the management of COVID-19 patients in intensive care units: recommendations and current evidence. Einstein (Sao Paulo). 2021; 19: eCE6419. (doi: 10.31744/einstein_journal/2021CE6419). PMID: 33787663; PMCID: PMC7971153.
12. Kamal M, Omirah MA, Hussein A, Saeed H. Assessment and characterisation of post-COVID-19 manifestations. Int J Clin Pract. 2021; 75(3): e13746. (doi: 10.1111/ijcp.13746). PMID: 32991035; PMCID: PMC7536922.

13. Giacalone M, Tovani-Palone MR, Marin L, Febbi M, Russano T, Giacalone A. Neurological and neuropsychiatric disorders associated with COVID-19. Part II: neuropsychiatric disorders and final consideration. Einstein (São Paulo). 2021; 19: eCE6464. (doi: 10.31744/einstein_journal/2021CE6464). PMID: 34730706 ; PMCID: PMC8528448. 